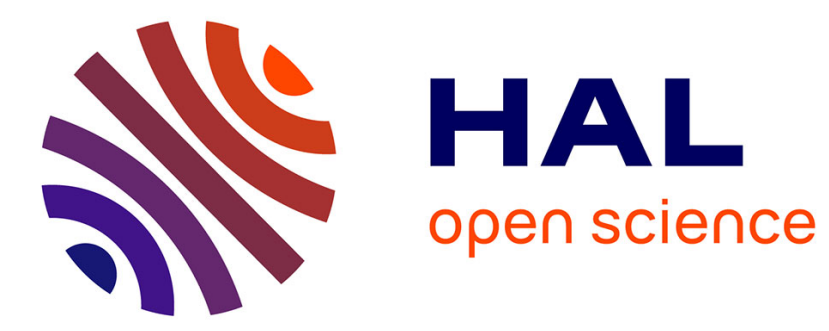

\title{
A hybrid semi-Lagrangian Vlasov code. II. Numerical simulation of chirped beatwave experiment
}

\author{
A. Ghizzo, Pierre Bertrand, J. Lebas, T. W. Johnston, M. Shoucri
}

\section{To cite this version:}

A. Ghizzo, Pierre Bertrand, J. Lebas, T. W. Johnston, M. Shoucri. A hybrid semi-Lagrangian Vlasov code. II. Numerical simulation of chirped beatwave experiment. Physics of Plasmas, 1998, 5 (11), pp.4041-4054. 10.1063/1.873126 . hal-01791849

\section{HAL Id: hal-01791849 \\ https://hal.univ-lorraine.fr/hal-01791849}

Submitted on 14 May 2018

HAL is a multi-disciplinary open access archive for the deposit and dissemination of scientific research documents, whether they are published or not. The documents may come from teaching and research institutions in France or abroad, or from public or private research centers.
L'archive ouverte pluridisciplinaire HAL, est destinée au dépôt et à la diffusion de documents scientifiques de niveau recherche, publiés ou non, émanant des établissements d'enseignement et de recherche français ou étrangers, des laboratoires publics ou privés. 


\section{A hybrid semi-Lagrangian Vlasov code. II. Numerical simulation of chirped beatwave experiment}

A. Ghizzo, P. Bertrand, J. Lebas, T. W. Johnston, and M. Shoucri

Citation: Physics of Plasmas 5, 4041 (1998); doi: 10.1063/1.873126

View online: https://doi.org/10.1063/1.873126

View Table of Contents: http://aip.scitation.org/toc/php/5/11

Published by the American Institute of Physics

\section{Articles you may be interested in}

Two-stage electron acceleration by simultaneous stimulated Raman backward and forward scattering Physics of Plasmas 2, 3115 (1995); 10.1063/1.871144

A hybrid Eulerian Vlasov code. I. Study of high-frequency beatwave experiment and Manley-Rowe action evolution in a finite causal system

Physics of Plasmas 3, 650 (1996); 10.1063/1.871892

Electron holes in phase space: What they are and why they matter

Physics of Plasmas 24, 055601 (2017); 10.1063/1.4976854

Stimulated forward Raman scattering of a laser in a magnetized plasma

Physics of Plasmas 14, 032109 (2007); 10.1063/1.2714499

A nonperiodic Euler-Vlasov code for the numerical simulation of laser-plasma beat wave acceleration and Raman scattering

Physics of Fluids B: Plasma Physics 2, 1028 (1990); 10.1063/1.859276

Stimulated Raman scattering: Action evolution and particle trapping via Euler-Vlasov fluid simulation

Physics of Fluids B: Plasma Physics 4, 2523 (1992); 10.1063/1.860168






\title{
A hybrid semi-Lagrangian Vlasov code. II. Numerical simulation of chirped beatwave experiment
}

\author{
A. Ghizzo, P. Bertrand, and J. Lebas \\ L.P.M.I.-C.N.R.S. UPRES-A 7040-Université Henri Poincaré, \\ BP 239, 54506 Vandoeuvre les Nancy cedex, France \\ T. W. Johnston \\ I.N.R.S. Energie, et Matériaux, C.P. 1020, Varennes, Québec J3X 1S2, Canada \\ M. Shoucri \\ Centre Canadien de Fusion Magnétique, Tokamak de Varennes, Varennes, Québec J3X 1S1, Canada
}

(Received 17 April 1998; accepted 23 June 1998)

\begin{abstract}
In this paper the results are reported of a set of numerical simulations applicable to the problem of particle acceleration by the beating of very high-frequency laser waves (the ratio of driver frequency to plasma frequency close to $\omega_{0} / \omega_{p}=30$ ), including for the first time beat frequency chirping (i.e., pump frequency linearly decreasing with time) at various rates in order to counteract relativistic electron detuning of the plasma oscillation frequency and the resulting Rosenbluth-Liu amplitude limit. This amplitude limit can indeed be evaded, giving extremely high acceleration gradients of more than $25 \mathrm{GeV} / \mathrm{m}$, for the case of numerical parameters relevant to the UCLA experiment (University of California at Los Angeles). A chirp rate that is too fast leads to a sudden loss of frequency lock and a subsequent decay of the driven electric field, in agreement with the behavior of a similarly driven relativistic Lagrangian Rosenbluth-Liu oscillator. Even at chirp rates somewhat below this limiting value a remarkably localized and extraordinarily intense (but as yet ill-understood) electric field structure is formed, one that is unlikely to be compatible with the general requirement for smooth and coherent acceleration. (c) 1998 American Institute of Physics. [S1070-664X(98)00310-3]
\end{abstract}

\section{INTRODUCTION}

In a previous paper ${ }^{1}$ we examined the space-time behavior of a plasma beatwave accelerator system using the newly developed Hilbert envelope extension (see Refs. 2 and 3) to the Vlasov fluid code we have developed and used before. The plasma beatwave accelerator concept of Tajima and Dawson ${ }^{4}$ has received much attention ${ }^{5-10}$ because of its promise of short accelerator lengths for a given energy gain due to the very large accelerating fields potentially available from driving the plasma resonance. However, as pointed out by Rosenbluth and Liu (see, for example, Ref. 7), well before the original proposal, the decrease of plasma frequency with an increase in wave amplitude (due to the oscillationvelocity-driven relativistic electron inertial mass increase) imposes an upper limit on the amplitude due to detuning from the driving ponderomotive at values often well below the plasma wave-breaking limit. This detuning limit may, in principle, be mitigated either (i) by a natural change of electron density from ionization ${ }^{8}$ or (ii) by using a monotonically decreasing driver frequency (a policy commonly termed frequency "chirping" for radar sources), i.e., the frequency difference between the driver-idler beatwave laser pair., ${ }^{9,10}$ The beatwave-driven local cold relativistic Lagrangian electron fluid oscillator model originated by Rosenbluth and $\mathrm{Liu}^{7}$ (hereafter usually abbreviated as RLO for the R-L Oscillator) is useful (see Refs. 7-9) for a basic understanding of the physics (and indeed provided encouragement to pursue further the feasibility of the chirped-drive concept).
In this paper we report an effort to go beyond the RLO model to include the space-time behavior necessary to evaluate an accelerator candidate. Of course, since the goal is to provide a series of well-organized particle bunches the essential accelerator problem requires at least the axial space dimension. While other saturation effects involving ion response such as the modulational instability (see, for instance, Ref. 11), are important for some systems, ${ }^{6}$ they are not important for the $\mathrm{CO}_{2} 10 \mu \mathrm{m}$ laser experiment at UCLA (University of California at Los Angeles; see, for instance, Ref. 5) and impossible to treat adequately in one spatial dimension and will not be discussed further here. In any case, because of the modest times (less than eighty plasma periods) for which we examine the problem, we have chosen to neglect ion motion completely and treat only electron motion (as in Refs. 1-5, 7-10) with nonuniformity only in the direction of laser and plasma wave propagation. In this paper we provide results obtained in exploring the effects of using frequency-chirped beatwave drive on a uniform plasma, with only axial spatial nonuniformity, with ion motion neglected.

Since, for usefully high beatwave phase velocities, the two laser frequencies must be far higher than the plasma frequency, it was necessary because of computer limitations, to avoid the burden of resolving these high frequencies and wavelengths. The method chosen to approximate these highfrequency beatwave effects was the Semi-Lagrangian Vlasov-Hilbert $(\mathrm{V}-\mathrm{H})$ complex envelope technique, as described and illustrated in our previous paper $^{1}$ (a paper to 
which we usually refer as Paper I or more simply as I), where both the details of the method and some no-chirp $V-H$ results are to be found.

The paper is organized in the following way. In Sec. II, to demonstrate the possibilities of the chirp concept, we present basic equations and numerical results of a highfrequency beatwave $H-V$ run, using beat frequency chirping at a relatively modest rate, but one which produced significant improvement in comparison with the no-chirp case. (By "improvement"' is meant that the wave amplitudes thus obtained proved to be considerably above the previous limit due to relativistic detuning, and thus gave significantly increased particle acceleration for the same length or the same time.) Next in Sec. III, various chirp rates are studied, both higher and lower than that of Sec. II, and the interpretation of the results is discussed in light of equivalent results (see, for instance, Refs. 8, 9) from the RLO model with linear chirp added. $^{8,9}$ (Here the emphasis is on the upper limit to useful chirp rates, as set by frequency unlocking.) Then, in Sec. IV, a chirped-drive numerical simulation is performed with parameters close to UCLA experiment (presented in Ref. 5), including the injection of an electron beam and the realistically slow rise of the electromagnetic drivers, together with a conservative beat frequency variation chirp rate. (While these last results do confirm the occurrence of strong particle acceleration induced by the introduction of the beatwave chirping, they also show an unexpected and undesired very strong field localization at late times.) Finally, Sec. V contains conclusions and some additional discussion.

\section{HIGH-FREQUENCY BEATWAVE NUMERICAL EXPERIMENT}

\section{A. Basic equations and chirped beatwave process}

As a device to avoid the Rosenbluth-Liu plasma wave amplitude limit due to the relativistic detuning of the beatwave-driven plasma wave, we introduce two electromagnetic waves (pump and idler or Stokes wave separated initially in frequency by the plasma frequency) with the pump frequency chirped linearly downward in time as follows, with the characteristic chirp time $T$ as the parameter whose effects are to be studied:

$$
\omega_{0}(t)=\omega_{0}(t=0)[1-2 t / T],
$$

and an idler wave of constant frequency-wavenumber $\left(\omega_{s}, k_{s}\right)$. Both incident electromagnetic waves, continuously injected at $x=0$, drive a forward-going longitudinal plasma wave with frequency and wavenumber $\left(\omega_{e}, k_{e}\right)$ according to the usual matching conditions:

$$
\omega_{0}(t)=\omega_{s}+\omega_{e} \text { and } k_{0}=k_{s}+k_{e} .
$$

In the Hilbert-Vlasov model (we have presented in Ref. 1) we replaced the high-frequency Maxwell's equations for the transverse waves by a two-wave complex envelope model coupled the complex spatial envelope $\rho_{e}$ of the electron plasma wave. These envelope equations (modified in order to take into account the beat frequency chirping) with spatio-temporal corrections are then

$$
\begin{aligned}
& {\left[\frac{\partial}{\partial t}+\nu_{g 0}(t-x / c) \frac{\partial}{\partial x}\right] A_{0}=\frac{-i \omega_{p}^{2}}{4 n_{0} \omega_{0}(t-x / c)} A_{s} \rho_{e},} \\
& {\left[\frac{\partial}{\partial t}+\nu_{g s} \frac{\partial}{\partial x}\right] A_{s}=\frac{-i \omega_{p}^{2}}{4 n_{0} \omega_{0}} A_{0} \rho_{e}^{*} .}
\end{aligned}
$$

Note that in this formulation no provision has been made for complex envelopes of either backward-propagating electromagnetic waves (implying no source for backward SRS), or for forward-propagating Stokes or anti-Stokes cascade waves. While the former could be done, it would have required spatial resolution appropriate for half the laser wavelength, rather less than the Debye length at our usual temperature, and the relevant plasma waves would have in any case been severely damped. The computer burden for including cascading into forward-propagating electromagnetic waves by adding envelope channels at frequencies separated by $\omega_{p}$, would have been much more modest (apart from the significant extra coding required), but we estimated that, at the drive amplitudes under consideration, frequency cascading was not likely to be important.

In our treatment, the pump frequency $\omega_{0}$ and therefore the linear pump group velocity $v_{g 0}\left(t^{\prime}\right)=\partial \omega / \partial k_{0}$ $=k_{0}\left(t^{\prime}\right) c^{2} / \omega_{0}\left(t^{\prime}\right)$ vary with $t^{\prime}=t-x / c$ [more accurately $\left.t^{\prime}=t-x / v_{g 0}(t)\right]$, due to the wave penetration in plasma. The boundary conditions are then given by

$$
A_{0}(x=0, t)=\frac{E_{0}}{\omega_{0} \sqrt{2}} \xi_{\text {profile }}(t) \exp i \frac{\omega_{0}(0) t^{2}}{T}
$$

and

$$
A_{0}(x=L, t)=0,
$$

and, for the idler wave,

$$
A_{s}(x=0, t)=\frac{E_{0}}{\omega_{s} \sqrt{2}} \xi_{\text {profile }}(t) \quad \text { and } \quad A_{s}(x=L, t)=0 .
$$

We have chosen $e E_{0} / m \omega_{p} c=3$ (so $e E_{0} / m \omega_{0, S} c \approx 1 / 10$ ). The initial growth in time $\tau$ of the electromagnetic waves to this constant value is modeled by the temporal profile $\xi_{\text {profile }}$ (with $\tau$ as the control parameter) as given by

$$
\xi_{\text {profile }}(t)=\left\{\begin{array}{l}
\sin ^{2}\left(\frac{\pi t}{2 \tau}\right), \quad \text { if } t \leqslant \tau, \\
1, \quad \text { if } t \geqslant \tau .
\end{array}\right.
$$

The coupling of these waves to the plasma wave envelope is arranged as follows, as described also in the companion paper I (i.e., Ref. 1). To couple from the envelope equations to the real Vlasov equation the real beatwave ponderomotive force is computed from the complex amplitude product $A_{0} A_{s}^{*}$ obtained from the equations for driver wave (pump and idler) complex envelopes. Going in the reverse sense, i.e., going from the real Vlasov charge density to the complex wave envelopes, Eqs. (3a), (3b), for the driver/idler electromagnetic waves, is done by generating the complex spatial envelope $\rho_{e}$ (and its complex conjugate $\rho_{e}^{*}$ ) with its phase referred to the uniform beatwave wavevector phase. It is this complex envelope which is obtained from the spatial 
Hilbert transform (see the discussion in paper I) of the real longitudinal plasma field used in the Vlasov equation.

\section{B. Numerical simulation of high-frequency beatwave without beat frequency chirping}

Because we wish to compare the numerical results of the chirped beatwave accelerator concept with the simpler case previously simulated in I, where the beat frequency was not chirped, we found it convenient to recall the more important features met in the earlier simulation. More details of the earlier simulation results, including numerical parameters have been given in Sec. V and table II of the companion paper (I).

The beat drive from two driver waves of frequency $\omega_{0}(t=0) \approx 30 \omega_{p}$ for the pump wave, and $\omega_{s} \approx 29 \omega_{p}$ for the idler wave was carried out with a relatively rapid (compared with experiment) amplitude rise time close to $50 \omega_{p}^{-1}$ for the electromagnetic waves (to reduce the computational burden). The ratio of the electron density to the critical density is then $n_{0} / n_{c r}=\left(\omega_{p} / \omega_{0}\right)^{2}=1.1 \times 10^{-2}$. The oscillation quiver momenta were $p_{\text {osc } 0} / m c=0.0708$ and $p_{\text {osc }} / m c=0.0734$ for the pump and idler waves, respectively. The results showed clearly the growth of an electron plasma wave with the expected normalized phase momentum of $p_{\phi} / m c=27.95$. As in the our earlier work, and in order to make a useful comparison with those results, the plasma is chosen with two electron temperatures, the majority $(95 \%)$ component with a relatively warm $15 \mathrm{keV}$ temperature (to reduce the Debyelength spatial resolution required) and a minority component $(5 \%)$ at $100 \mathrm{keV}$ to provide sufficient electron density in phase space for convenience in diagnosing electron acceleration features.

Figure 1 illustrates the propagation and growth of the (real) plasma electric field. Very early in time, as shown in Fig. 1 at time $t \omega_{p}=200$, the spatial growth of the oscillating electric field amplitude is indeed linear (i.e., secular), as predicted by theory for a resonant excitation. [Note for reference that, in our usual electric field units (which are normalized to $\left.m \omega_{p} c / e\right), e E / m \omega_{p} c$ is also very nearly equal to the density modulation $\delta n / n_{0}=\left(k_{e} c / \omega_{p}\right)\left(e E / m \omega_{p} c\right)$ since we have $k_{e} c / \omega_{p}=1.0479$.] The growth slows down later as relativistic detuning makes itself manifest.

The Rosenbluth-Liu expression for the normalized spatial growth rate in our normalization is given by $d\left(e E / m \omega_{p}^{2}\right) / d x=-v_{o s c 0} v_{o s c} / 4 v_{\phi}^{2}$, where $v_{o s c 0}$ and $v_{o s c S}$ are the "quiver velocities" (actually quiver momenta divided by electron mass, i.e., $p_{\text {osc }} / m$ ) of the pump and idler wave, respectively. With the values of these quantities given in table II of paper I, we obtain then $d\left(e E / m \omega_{p}^{2}\right) / d x$ $=-1.296 \times 10^{-3}$. In excellent agreement with this simple estimate, the numerical value of the envelope slope yields $-1.30 \times 10^{-3}$. A limit on the longitudinal electric field is clearly visible by the time $t \omega_{p}=300$, leading to the appearance of a modulation structure of length $\omega_{p} l / c \approx 320 \approx 53 \lambda_{e}$ $\approx 1530 \lambda_{0}$ (which means that $l \approx 16 \mathrm{~mm}$ for a $\mathrm{CO}_{2}$ laser of wavelength $\lambda_{0}=10.59 \mu \mathrm{m}$ ). (However, by the time $t \omega_{p}$ $=500$, the plasma wave envelope structure to the left varies much more rapidly in space.) It is also natural to compare the


FIG. 1. Growth of the normalized driven plasma wave electric field without beat frequency chirping. Limiting of the field due relativistic detuning is clearly visible by the time $t \omega_{p}=300$, leading to the appearance of a modulation structure front end resembling the temporal behavior of an RLO system with a constant unchirped driver. However, the behavior close to entry is less transparent.

saturation level of the electric field with the prediction related to be relativistic detuning. Using a simple Lagrangian cold electron fluid description and assuming a constant amplitude for the two pump waves (see also Sec. III), the Rosenbluth-Liu ${ }^{7}$ limit amplitude of the electric field takes the following value in the present case:

$$
\frac{e E_{R L}}{m \omega_{p} c}=\left(\frac{\omega_{p}}{k_{e} c}\right)\left(\frac{8}{3} \frac{c^{2} v_{o s c 0} v_{o s c S}}{v_{\phi}^{4}}\right)^{1 / 3}=0.229,
$$

while the $\mathrm{H}-\mathrm{V}$ code gives a weaker saturation value of 0.145 .

As pointed out in Sec V A of paper I, significant action transfer occurs and this always involves a change of global phase, a phenomenon beyond the scope of the oscillator model. While this may play a role, it was found in an earlier ${ }^{1}$ discussion (in Sec. V A and Figs. 5-7 of I), that a dissipationless coupled-mode model including the plasma wave group velocity does give the same value as the RosenbluthLiu oscillator ${ }^{7}$ (=RLO). Hence, as also discussed earlier, ${ }^{1}$ the difference seems to reside in the difference in energy supplied to the accelerated electrons from the $100 \mathrm{keV}$ tail. It appears that even though the electrons actually trapped were a negligible fraction of the energies involved, some interaction with the untrapped hot tail accounted for the difference. In support of this point, a comparison of this result with that given earlier ${ }^{1}$ for a much colder $(1 \mathrm{keV})$ plasma showed behavior rather closer to the oscillator model (where temperature plays no role). At this point all that one can immediately infer from this is that the tail temperature clearly plays a significant (but unclear) role in mediating the action transfer. For most of the work of this paper, we have kept the same distribution function for two reasons: (i) ease of com- 

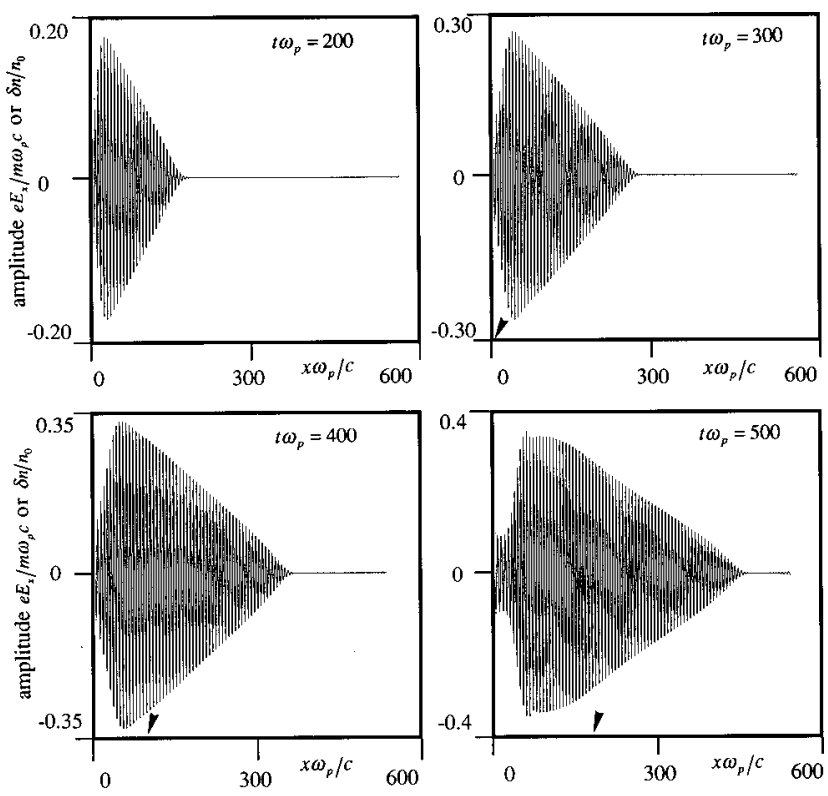

FIG. 2. Time evolution of the longitudinal electric field with a beatwave frequency chirping time of $T \omega_{p}=5 \times 10^{5}$. The electric field is seen to grow secularly with time (i.e., it is linearly ramped in space) until $t \omega_{p}=500$, without the RLO leading structure of Fig. 1.

parison with the previous work and (ii) electron loading must in any case be eventually included in evaluating an accelerator.

\section{A conservatively "chirped" laser beatwave}

\section{Evolution of plasma wave and electromagnetic driver waves}

To test the idea that a chirped beatwave accelerator could provide significantly stronger plasma wave amplitudes, the pump system discussed in the previous section (and in I) (with $p_{o s c} / m c=0.07$ ) is employed but now with a chirped frequency. Here the compensation of the relativistic frequency shift is achieved by an appropriate value for the linear time-rate change of one driver component (the pump is our choice) frequency in time. The chirped frequency used is given by Eq. (1) with a starting value of $\omega_{o}(t=0)$ $=30.01 \omega_{p}$ and a chirp time of $T \omega_{p}=5 \times 10^{5}$, leading at a time of $t \omega_{p}=500$ to a difference frequency of $\Delta \omega=\omega_{0}(t)$ $-\omega_{0}(t=0)=0.06 \omega_{p}$.

We first discuss the plasma wave amplitude growth. In Fig. 2 (which should be compared with Fig. 1) one can see at different times the slowly oscillating plasma field. Because the pump frequency is now slowly decreasing in time, the plasma oscillation are able to stay locked to the driving force, exhibiting linear (i.e., secular) growth for a much longer time, at least until $t \omega_{p}=400$. The wave amplitude behavior is in significant contrast to the behavior with the unchirped driver (as shown in Fig. 1), the wave amplitude with chirp thus reaching much higher values (about 0.35 instead of 0.14 , or about 2.5 times higher). Clearly (at least until this time) there is much to be said for the chirp concept as a way of producing stronger waves in the same time and plasma length with the same laser intensities. The beatwave sources (incident from the left) exhibit the usual ${ }^{1}$ action transfer between the pump, the idler and plasma wave. Significant pump depletion (not shown here) was observed by the end of these simulations. Hence one may expect significant differences here from the simple RLO model, where space-time action transfer effects cannot be realistically included. The observed spatial secular growth rate yields the value $-1.294 \times 10^{-3}$ (measured at time $\left.t \omega_{p}=200\right)$ in good agreement with the predicted value of $\left(e / m \omega_{p}^{2}\right)(d E / d x)$ $=-\left(v_{o s c 0} v_{o s c S}\right) / 4 v_{\phi}^{2}$, when relativistic detuning is neglected.

In spite of the limiting behavior seen at late times after $t \omega_{p}=400$, this relatively small beatwave chirping rate $\left(T \omega_{p}=5 \times 10^{5}\right)$ of the higher driver wave has allowed the system to drive the plasma wave up to an amplitude that is not only (see Fig. 2) about 2.5 times the value attained without chirping (Fig. 1), but also 1.5 times higher than the theoretical Rosenbluth-Liu limit. The maximum normalized electric field amplitude given by the code of about 0.34 does agree with the wave-breaking amplitude for a nonrelativistic waterbag hot plasma as given by Coffey: ${ }^{12}$

$$
\frac{e E_{W K}}{m \omega_{p} c}=\frac{v_{\varphi}}{c}\left[1-\frac{8}{3} \mu^{1 / 4}+2 \sqrt{\mu}-\frac{\mu}{3}\right]^{1 / 2}(\approx 0.33),
$$

where the parameter $\mu$ is defined by $\mu=3 v_{t h}^{2} / v_{\phi}^{2}$ $(=0.0882)$. However, this value is far less than the theoretical value for relativistic waves given by Sheng and Meyerter-Vehn in Ref. 13, who also reconciled earlier results (which they discuss) for a fluid plasma. Among other results, they give an approximation that is valid for this case (low temperature and near-light phase velocity) and this is as follows:

$$
\frac{e E_{W B \mathrm{rel}}}{m \omega_{p} c}=\left(\frac{4}{9 \mu}\right)^{1 / 4}(\approx 1.5) .
$$

Since the wave is relativistic, the previous equation should apply, so one can conclude that the normalized electric field value reached in the $\mathrm{H}-\mathrm{V}$ simulation (0.34) is not being limited by relativistic wave-breaking and that the agreement with Coffey's nonrelativistic result seems to be a coincidence.

\section{Phase space representation}

The fast electron generation is illustrated in Fig. 3 by a plot of a part of phase space covering $75<x \omega_{p} / c<225$ and positive $p_{x} / m c$ values up to 60 at the end of the simulation time $t \omega_{p}=500$. [Since particles are accelerated with positive values in the vicinity of the phase momentum of the electron plasma wave $p_{\phi} / m c(=27.95)$, only the upper half of the normalized $x-p_{x}$ phase space plane has been represented, but only for values of the distribution function significantly greater than zero and less than $10^{-5}$, above which value the distribution function is truncated.] As in our earlier papers the superimposed curves show the calculated separatrix space-momentum locus for marginally trapped electrons in the electron plasma wave at the current field values.

From Fig. 3 one can clearly see values for $p_{\max } / m c$ of up to 60 or so have been obtained, using the chirp rate of Sec. II C 1 (corresponding to a chirp time of $T \omega_{p}=5 \times 10^{5}$ ) and 

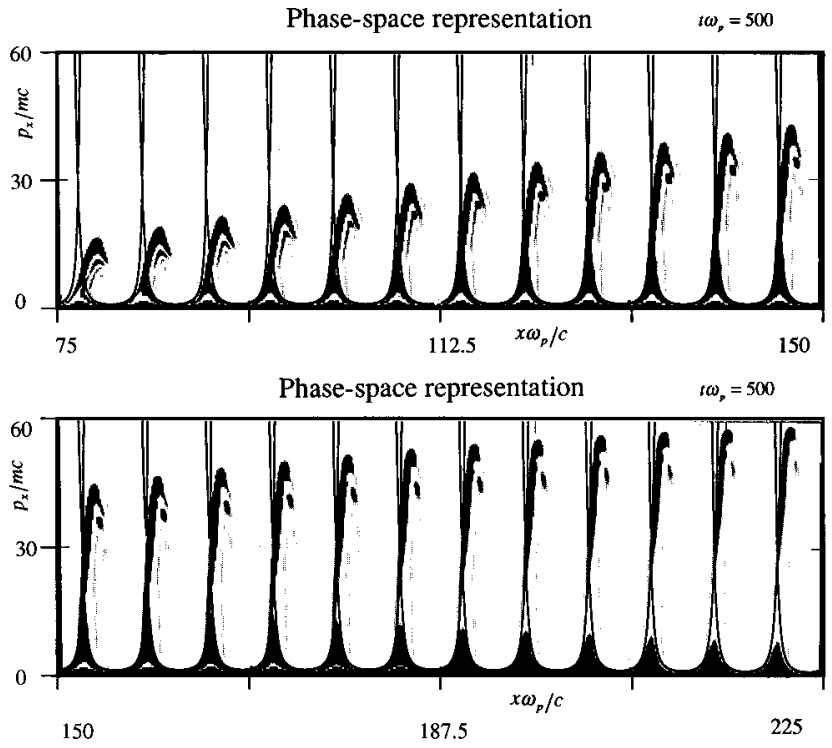

FIG. 3. A plot of a region of normalized $x-p_{x}$ phase space of the electron distribution function at the end of the chirped-driver simulation of Fig. 2, for $t \omega_{p}=500$, showing clearly the steady particle acceleration due to pump frequency chirping. As in Ref. 1, the solid lines show the ideal steady-state separatrices of marginally trapped electrons for the instantaneous plasma wave electric field

the same idler as before. In comparison with the identical system without chirp, for which (as shown in Paper I) the maximum value of $p_{\max } / m c$ was only about 30 (i.e., just about $p_{\text {phase }} / m c$ ), the maximum accelerated particle energy was increased by chirping by a factor of about 2 , with maximum energies going from $15 \mathrm{MeV}$ to $30 \mathrm{MeV}$ well above the phase value but well short of the ultimate value were there no limits on plasma length or simulation time.

To emphasize the connection with the UCLA experiment, ${ }^{9}$ which uses a driver $\mathrm{CO}_{2}$ laser wavelength of $\lambda_{0}=10.275 \mu \mathrm{m}$ (and hence a plasma wavelength of 30 times this), we will also indicate the relevant distances in ordinary units for that case. As a first example, the phase space data of Fig. 3 also exhibit clearly the fact that the acceleration mechanism produces tight electron bunches which are typically submillimeter (about $0.25 \mathrm{~mm}$ ) in length when scaled to those of the UCLA experiment.

To show how the acceleration of the electrons in a given phase-space "bucket" evolves, a moving window display was constructed, with length $l=3 \lambda_{e}$ (about $18 c / \omega_{p}$, corresponding to $1 \mathrm{~mm}$ in the UCLA case), and effective velocity chosen equal to the linear phase velocity value (very close of the light velocity). Some of those results are shown in Fig. 4. (The spatial location of this moving window throughout the simulation is indicated in Fig. 2 by an arrow on the longitudinal electric field snapshot plots.) The particle population of the moving window experiences a nearly constant acceleration field during their motion. Thus, at time $t \omega_{p}=500$, some electrons located between $200<x \omega_{p} / c<220$ have gained at least $30 \mathrm{MeV}$ in traversing $200 c / \omega_{p}$ (corresponding to about $950 \lambda_{0}$ ) or a length of about 1 centimeter (at UCLA) plasma wave region, which corresponds to an average accelerating field of more than $2.8 \mathrm{GeV} / \mathrm{m}$.
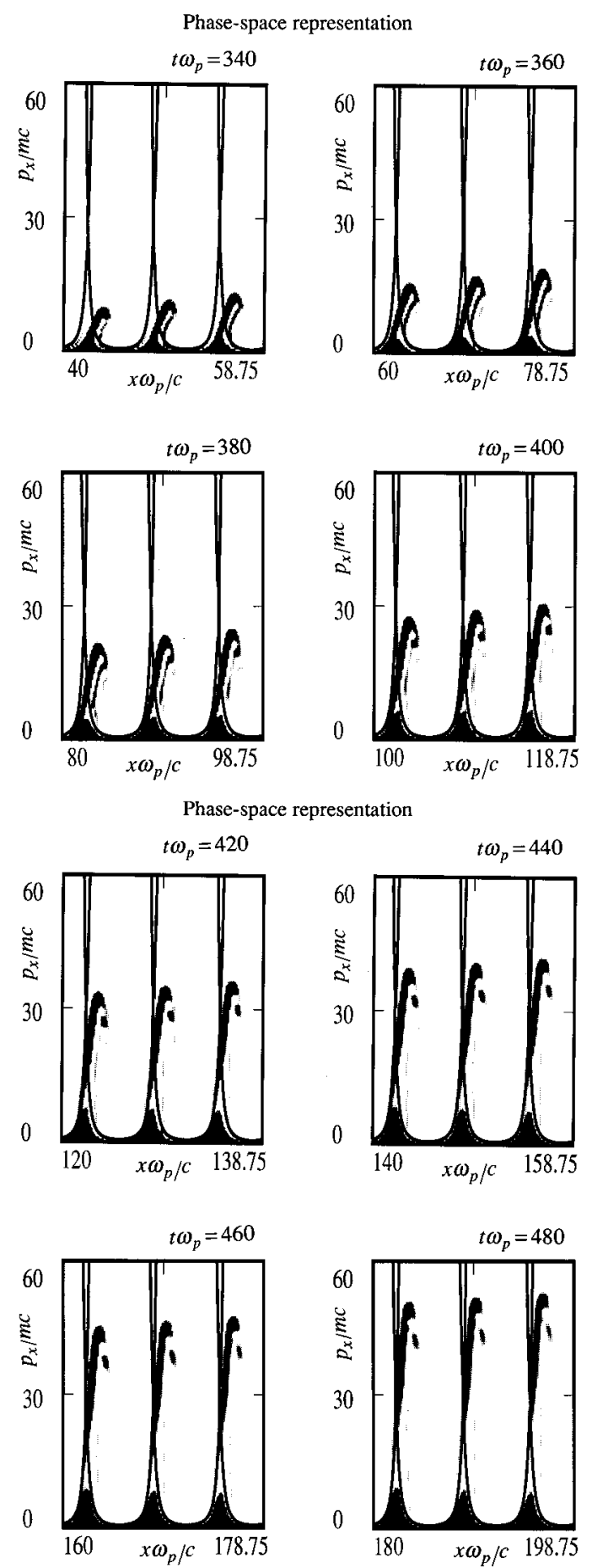

FIG. 4. From the same simulation as for Figs. 2 and 3, the corresponding phase-space representation of a three-wavelength window moving at the light velocity for normalized times from 340 to 480 . The trapping structures are only slowly varying in the wave frame giving a time history of the same population of trapped particles. These trapped particles experience a fairly constant acceleration gradient during their motion.

\section{Manley-Rowe action evolution}

For a better understanding of the difference in the behavior of the chirped drive considered here, it is instructive to compare the evolution of the action density of the different modes involved in the parametric instability. In the companion paper (I) we have derived the Manley-Rowe relations in a finite causal system from a three-wave (or four-wave if the 
anti-Stokes mode is included) model. It is useful—especially as regards the interpretation of simulation results-to recapitulate briefly, following our earlier work (given in Ref. 11) the form taken by the Manley-Rowe relations in the present study.

The sources in this space-time system are the net action fluxes injected and lost (in this case the latter are zero since in the time of the simulation the exit boundary is not reached) from the pump and idler waves. These net normalized action fluxes at the boundaries (the "surface" terms) are denoted by $F_{i}$ [defined by relation $F_{i}$ $\left.=a_{i} a_{i}^{*} v_{g i} \omega_{p} /\left(n_{0} m c^{3}\right)=S_{i} v_{g i} / c\right]$. Here $S_{i}=a_{i} a_{i}^{*} \omega_{p} /$ $n_{0} m c^{2}$ is the normalized action density and $\left(a_{i} a_{i}^{*}\right)$ represents the unnormalized action density (see Ref. 3 for more details). Thus, $F_{i}$ represents the normalized net action (photon) flux penetrating the plasma slab at $x=0$ less that leaving at $X=L$ (which has not happened in these simulations), with $i$ now denoting either 0 or $s$, for the pump and idler, respectively. Defining the normalized total action (equivalent to the number of photons or plasmons per area unit in the plasma slab of length $L$ by $N_{i}=\int_{0}^{L} S_{i} d X$, where $X$ is $x \omega_{p} / c$, with $i$ now denotes " 0 " for the pump wave, " $s$ " for the idler wave, and finally " $e$ " for the plasma wave, the Manley relations can then be written in the form

$$
\begin{aligned}
& \frac{d}{d t}\left(N_{0}+N_{s}\right)=F_{0}+F_{s}, \\
& \frac{d}{d t}\left(N_{0}+N_{e}\right)+2 \mu N_{e}=F_{0}, \\
& \frac{d}{d t}\left(N_{e}+N_{s}\right)+2 \mu N_{e}=-F_{s} .
\end{aligned}
$$

We obtain then the form useful for checking action transfer to electrons (where $\mu$ is a phenomenological damping factor):

$$
2 \mu N_{e}=F_{0}-\frac{d}{d t}\left(N_{0}+N_{s}\right) .
$$

In the lossless Vlasov plasma, with a single plasma wave being responsible for the energy transferred to fast electrons, we have hypothesized that the kinetic (relativistic) energy of all electrons above the lower momentum boundary of the wave instantaneous separatrix would account for the energy missing and (on division by the electron plasma wave frequency and $\hbar$ ) for the corresponding missing "mean number of plasmons of wave-particle interaction (per unit area). Thus, defining

$$
N_{W P}=\frac{m c^{2}}{\hbar \omega_{e}} \int_{0}^{L} d x \int_{P \text { low }}^{+\infty} d p_{x}(\gamma-1) f\left(x, p_{x}, t\right),
$$

Eqs. (6a), (6b), and (6c) can now be rewritten in the following form (with $\tau$ as the normalized time variable $\omega_{p} t$ ):

$$
\begin{aligned}
& \frac{d}{d \tau}\left(N_{0}+N_{s}\right)=F_{0}+F_{s}, \\
& \frac{d}{d \tau}\left(N_{0}+N_{e}+N_{W P}\right)=F_{0},
\end{aligned}
$$

$$
\frac{d}{d \tau}\left(N_{e}+N_{W P}-N_{s}\right)=-F_{s} .
$$

Note that Eq. (7a) generalizes, to the case of an open system with incoming flux, the first (electromagnetic) invariant sum of this Manley-Rowe system as met in an earlier study $^{2,3}$ of a periodic system in the usual form [i.e., $C_{s}(t)$ $\left.=\left|a_{0}\right|^{2}+\left|a_{s}\right|^{2}=C_{s}(t=0)\right]$. While for the periodic system conservation of the previous sum action $C_{s}(t)$ means photon conservation, here we have to take into account the photon injection in the open system. For the time of our simulation, while considerable action flux has been injected, no flux has yet left the system yet, so conservation is indicated by linear growth for a uniform beatwave.

Since the plasma wave interacts nonlinearly with both pump and idler wave as well as with the trapped and nearly trapped electrons, we have to consider the action sum of pump, plasma wave, and action transferred to fast electrons described in relation (7b). In addition to the total electron plasma wave action $N_{e}$ we also have as before used the trapping separatrix boundary (see, for instance, Ref. 3) to define the total transferred action (or equivalently) the normalized total equivalent number of plasmons $N_{W P}$ transferred to trapped electrons due to wave-particle interactions using relation (21) of paper I. As we emphasized in Sec. III of the paper I, both the electron plasma wave action $\left(\right.$ as $\left.N_{e}\right)$ and the action $N_{W P}$ transferred from the plasma wave to fast electrons are included. [We have defined fast electrons as those which are above the lower boundary of the separatrix and action sum $N_{W P}$ as being their energy sum divided by the associated wave (i.e., plasma) frequency.]

For the normalized chirp time of $5 \times 10^{5}$, the corresponding time evolution of different quantities described in Eqs. (7a) $\left(N_{0}, N_{S}\right.$, and $\left.N_{e m}\right)$ and (7b) $\left(N_{0}, N_{e}, N_{W P}\right.$, and $N_{2}$ ) are represented in Fig. 5(a) and Fig. 5(b), respectively, as solid curves. In order to make a comparison with the case where the pump wave is not chirped, we have superimposed as dashed curves in each figure the corresponding curves [shown in Fig. 7 of paper (I)] for the zero chirp case (i.e., for $\left.T \omega_{p}=\infty\right)$.

The temporal behavior of the electromagnetic actions $N_{0}, N_{s}$, and their sum $N_{e m}\left(=N_{0}+N_{s}\right)$ is first displayed in Fig. 5(a), where one can compare the effectiveness of the mode conversion from pump to Stokes (idler) wave with associated the plasma wave in Fig. 5(b), as shown by the increasing gap between $N_{s}$ and $N_{0}$. The evolution of $N_{e m}$ is identical for both cases with and without chirp, as should be the case since this sum is really a check on the fact that there is no action loss for the electromagnetic waves. At early times (i.e., until $t \omega_{p}=200$ or so, before relativistic detuning can play a role in either case) the behavior for the individual $N_{s}$ and $N_{0}$ components is also quite similar in both cases. Beyond that time in the chirped case there is little increase in total injected pump action $N_{0}$ because this slow chirp is nonetheless so effective that it leads to nearly complete conversion of the recently injected pump action to Stokes action. [This is why the Stokes (idler) total action $N_{s}$ evolves linearly with time, at late times, with a slope of $F_{0}+F_{s}$, the same as the slope of the $N_{e m}$ sum.] Because of the decou- 




FIG. 5. For the same simulation as for Figs. 2-4, this is the time evolution of the total "number" of pump "photons" (actually normalized wave action) $N_{0}$ (per unit area), of idler "photons" (action) $N_{s}$. Also shown are the corresponding number of plasmons (plasma wave action) $N_{e}$, and the number of plasmons $N_{W P}$ converted to particle acceleration due to waveparticle interactions. Also shown are their respective sums: (a) $N_{e m}=N_{0}$ $+N_{S}$; (b) $N_{2}=N_{0}+N_{e}+N_{W P}$. The dotted lines correspond to numerical results presented in Ref. 1 without pump frequency chirping. A slow pump frequency variation leads to an effective saturation of the pump wave action, indicating that each photon lately injected into the plasma decays in a scattered photon and plasmon.

pling due to relativistic detuning in the unchirped case at late times, the overall conversion rate from pump to idler (Stokes) action (i.e., the slope of the $N_{s}$ curve) for the chirped case is about double that of the case without chirp.

To examine the plasma wave and particle acceleration behavior Fig. 5(b) should be consulted next, where the results are shown for $N_{0}, N_{e}, N_{W P}$, and their sum, $N_{2}$. As one should expect from the results of Fig. 5(a), where chirp has clearly improved the electromagnetic conversion from the pump, one obtains a much higher level of plasma wave action $N_{e}$. Indeed, at the end of the simulation the total plasma wave action is about 6 times that without chirp, and this in spite of the fact that about a third of that was already transferred to trapped fast electrons, meaning that the total transfer rate to plasma waves was actually about 8 times larger with the use of chirp. The fast electron component itself (extracted from the $100 \mathrm{keV}$ tail), which was relatively
TABLE I. A comparison of numerical and analytic results of normalized photon flux obtained from the data of the mean number of photons and plasmons in the box simulation (Manley-Rowe action evolution).

\begin{tabular}{llcc}
\hline \hline \multicolumn{1}{c}{ Action sum } & Photon flux & Theoretical value & Numerical value \\
\hline$N_{0}+N_{e}+N_{W P}$ & $F_{0}$ & 0.075 & 0.072 \\
$-N_{S}+N_{e}+N_{W P}$ & $F_{S}$ & 0.077 & 0.078 \\
$N_{0}+N_{S}$ & $F_{0}+F_{S}$ & 0.1526 & 0.1531 \\
\hline \hline
\end{tabular}

negligible without chirp, was enormously increased. With chirp, the rate of conversion to fast electrons at the end of the simulation was more than half the maximum action transfer rate possible (which is that of electromagnetic action transfer).

By all the measures just discussed [the conversion of energy from pump to idler in Fig. 5(a) and from pump to plasma waves and to fast electrons in Fig. 5(b)], it is clear that application of this relatively conservative chirp rate has dramatically improved the efficiency and speed of transfer from the beatwave source to plasma waves and fast electrons.

The various final action sums, as presented in Table I, are in good agreement with the expected values.

\section{COMPARISON WITH THE ROSENBLUTH-LIU RELATIVISTIC LAGRANGE OSCILLATOR PREDICTION}

The chirped beatwave concept has been explored previously using the relativistic Rosenbluth-Liu oscillator, ${ }^{7-10}$ which in normalized (to $c / \omega_{p}$ ) Lagrangian coordinates $\xi$ has the following form:

$$
\gamma^{3} \frac{d^{2} \xi}{\omega_{p}^{2} d t^{2}}+\xi=\frac{\epsilon(t)}{2} \sin \left(\xi_{0}-\psi(t)\right)
$$

where $\xi$ and $\xi_{0}$ are the Lagrangian coordinate and its reference value, respectively, of an oscillating electron cold fluid planar element whose position $x$ is related to the Lagrangian variable $\xi$ by $x \omega_{p} / c=\xi+\xi_{0}, \xi_{0}$ being its equilibrium point (usually zero when one speaks of only one oscillator at a time as here) and $\epsilon=v_{o s c 0} v_{o s c S} / c^{2}$ is the (small) beatwave source term. The beat frequency chirping if present is manifest when $\psi(t)$ has a form other than $\left(\omega_{0}-\omega_{s}\right) t$ where $\omega_{0}$ and $\omega_{s}$ are constants. With nonzero chirp the general expression for the phase $\psi$ of the driving force becomes

$$
\psi(t)=\int_{0}^{t} d t\left(\omega_{0}(t)-\omega_{s}\right)
$$

Using appropriately slowly chirped beatwave pumping in numerical integration of Eq. (8), the amplitude of plasma wave has been observed to increase dramatically (even to nominal wave-breaking levels (that is, to where $\left\langle\xi^{2}\right\rangle^{1 / 2}$ becomes comparable to 1 ), because the phase lock be maintained for a much longer time than without chirp. Below what proves to be a critical value for losing phase lock with the driver pair, the penalty for too low a chirp rate is simply inefficiency, in that it takes longer to reach the same value for $\xi$, and usually with more oscillations in $\xi$ as seen in the results shown later (in Fig. 6). 


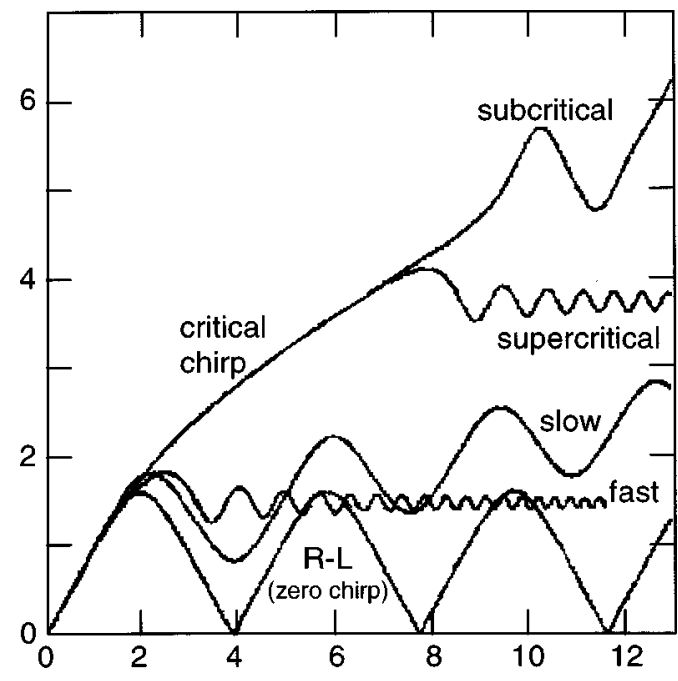

FIG. 6. Lagrangian Rosenbluth-Liu oscillator (RLO) results for various chirp rates (as discussed in Sec. III). In the Mora normalization presented in Ref. 14 the subcritical chirp value is 1.899380261 , while the supercritical chirp value is 1.899380801 . The slow chirp value is 0.40469 , while the fast chirp value is 2.02346 . (The actual value used for $\epsilon / 2$, the constant driver was $3.69 \times 10^{-3}$, but it has been checked that Mora normalization holds well and that the normalized result is indeed independent of driver strength for drivers whose strength does not approach one.)

When the chirp rate is too large the plasma remains phase-locked to the chirping driver but for only a limited time, at which point there occurs a sudden loss of phase and frequency lock, followed by a transition to a combination of a much lower-amplitude source-driven state together with a free oscillation continuing at the oscillation frequency when the frequency unlocking occurred. In previous work (as yet published only in abstracts ${ }^{9}$ ) the critical linear chirp value for losing phase lock was determined numerically for the linear chirp rate for a beatwave driver with a constant amplitude in the Lagrangian oscillator approximation. The numerically determined normalized critical chirp rate $F_{\text {crit }}^{\prime}$ (in the normalization used by Mora given in Ref. 14) was determined to be 1.89938. (This value is for a linear chirp rate and a constant amplitude drive. Other drive or chirp forms such as that of Deutsch et al. ${ }^{10}$ would require their own analysis. Laser experts would find a significant linear chirp rate challenge enough without requiring other complications such as a specific form such as an exponential.) In the units used here this normalized rate translates to $\omega_{p} T_{\text {crit }}=2\left(\omega / \omega_{p}\right) R^{2} / 1.89938$, with $R$ defined as $(256 / 3)^{1 / 3}\left(c^{2} / v_{o s c 0} v_{o s c S}\right)^{2 / 3}$. In the case at hand this becomes $\omega_{p} T_{\text {crit }}=7.3568 \times 10^{5}$. Although the RLO model is highly idealized, it was decided to explore the effect of different chirp rates and comparing the results with the chirped RLO results.

In Fig. 6 are shown some numerical results (as yet unpublished elsewhere) for the Lagrangian oscillator using the full normalization as introduced by Mora ${ }^{14}$ for a constant amplitude drive. The results for various chirp rates are shown both above and below the maximum critical linear chirp rate $F_{\text {crit }}^{\prime}$ at which oscillator locking to the drive is suddenly lost. The various rates are labeled as follows: the two cases close to critical are labeled as "supercritical" for the chirp rate slightly above critical, as "subcritical" for the case slightly
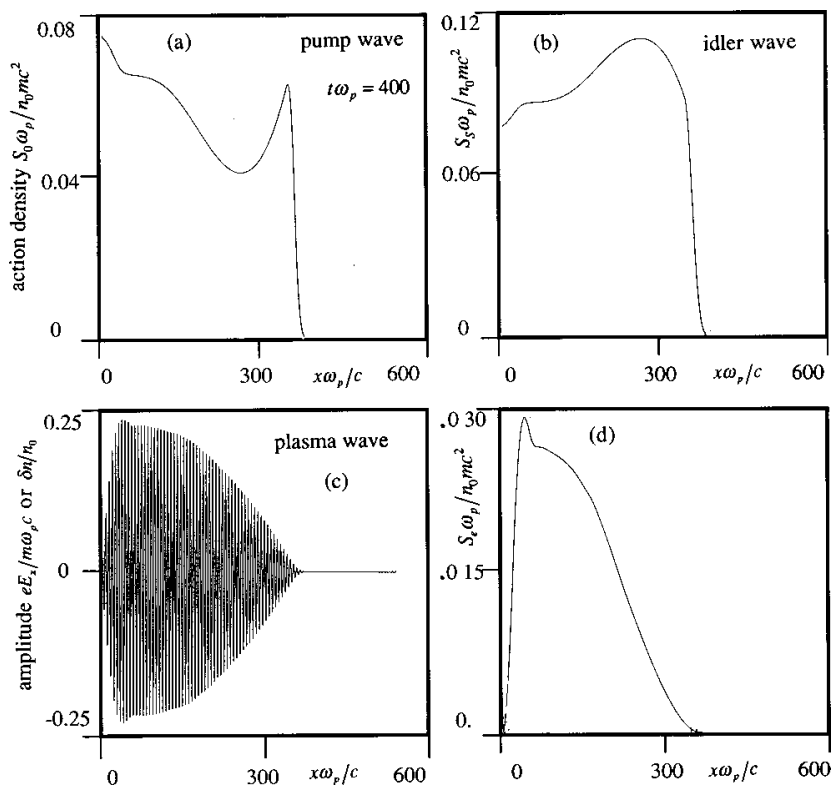

FIG. 7. This simulation corresponds to a longer chirp time (i.e., slower chirp) compared to that of Figs. 2-5, the chirp time being $T \omega_{p}=8.5$ $\times 10^{5}$. Shown at time $t \omega_{p}=400$, are the spatial behavior of the pump wave envelope in (a), of the idler wave in (b), and the corresponding plasma electric field is shown in (c), together with its envelope in (d) in density action units. [Note the small bump near the left-hand side in (c) and (d).]

below critical, and as "fast" for the chirp rate well above critical and "slow" for the case of a rather slow chirp. For reference the well-known unchirped periodic result of Rosenbluth and Liu (see Ref. 7) is also shown (labeled as " $R-L$ "). After frequency lock is lost for chirp rates above critical (i.e., "supercritical" and "fast" cases), the observed amplitude oscillations at steadily increasing frequency, have been determined as being due to the beating of a large but unforced oscillation still at the frequency at which frequency lock was lost, and the smaller-amplitude phase-locked forced response possible at the current value of the beatwave drive frequency.

\section{A. Chirped beatwave experiment in the case of a too slow chirp rate}

To explore the effects of varying the chirp rate, we begin by using a chirp rate of about 0.6 that of Sec. II, with a longer chirp time of $T \omega_{p}=8.5 \times 10^{5}$ (as compared with the earlier value of $\left.T \omega_{p}=5 \times 10^{5}\right)$. A numerical simulation is performed with this slower chirp rate and the results are shown in Fig. 7 (at a convenient common time $t \omega_{p}=400$ ). The results shown in Fig. 7 include the spatial behavior of both of the beatwave driver's electromagnetic component envelopes [the pump wave in Fig. 7(a) and idler wave in Fig. $7(b)]$ in normalized density action units, as usual), together with the oscillating longitudinal electric field, shown in Fig. 7 (c) (normalized as usual to $m \omega_{p} c / e$ ), with the corresponding action envelope plotted in Fig. 7(d).

The envelopes of driver modes resemble the general features, but with less marked variation, of those observed in the previous case of Fig. 2 with a stronger chirp rate. There are, however, some noticeable differences in the shape of the 


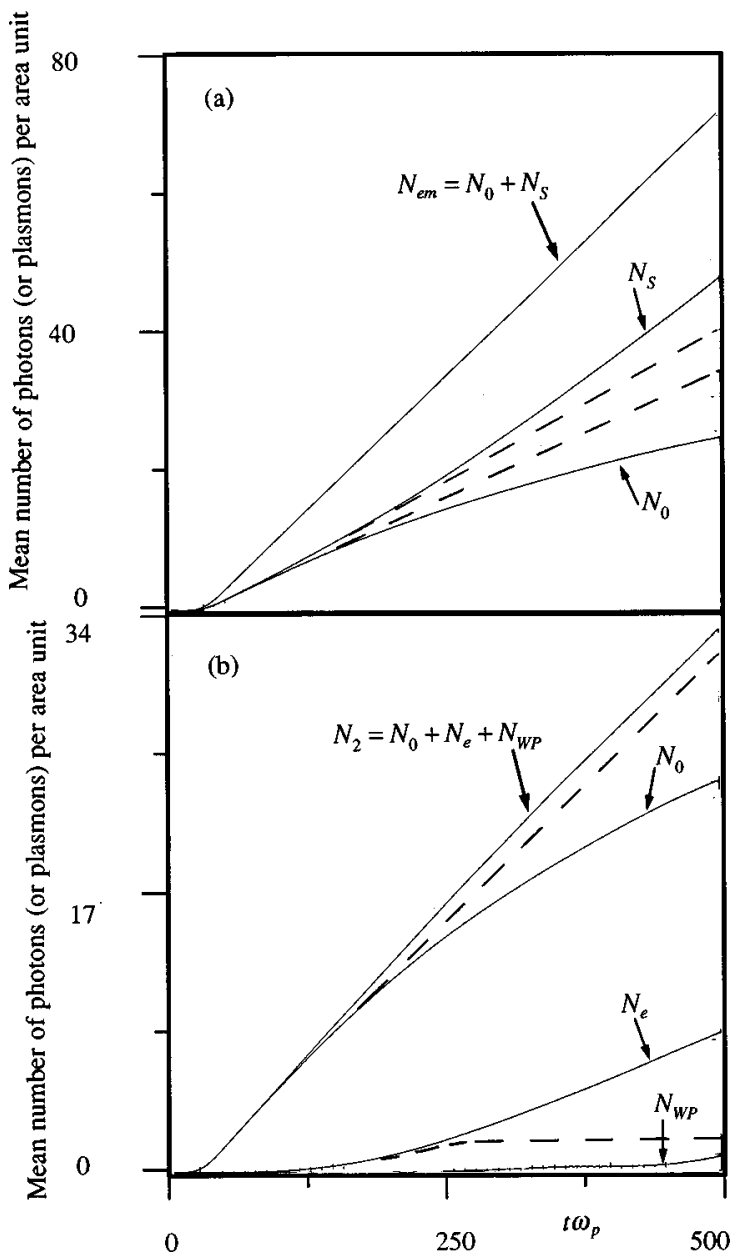

FIG. 8. The same action and action-sum diagnostic as for Fig. 5 but now for the case of Fig. 7, with its somewhat slower chirp $\left(T \omega_{p}=8.5 \times 10^{5}\right)$. As expected all the indicators of efficient acceleration are reduced with respect to Fig. 5.

envelope of the plasma wave. The plasma wave in Fig. 7(c) shown at $t \omega_{p}=400$ has already reached its maximum level, which is lower (at a normalized value of about 0.23 as compared with the earlier value of 0.35) than that of Fig. 2 at the same time. (Of course the value is still much greater than the value of 0.17 in the no-chirp case of Fig. 1.) Examining the electric field results at the same time $\left(t \omega_{p}=400\right)$ for the three cases, one can see clearly that, while there is no sign yet of leveling off in plasma wave field amplitude for the fastest chirp case, Fig. 2, the typical no-chirp oscillation of the type discussed by Rosenbluth and Liu (see Ref. 7) and by others (see Refs. 8, 9) is clearly evident in Fig. 1. The present case is in all respects intermediate between these two, already showing signs of leveling off and likely to begin to show the typical "slow chirp" oscillatory behavior of Fig. 6.

Repeating, in Fig. 8, the integrated action comparisons between this weak chirp case and the no-chirp case (I) that were carried out in Fig. 5 for the significant chirp and nochirp case, we can see again that this weak-chirp case is intermediate between the two. To begin with, using Fig. 8(a) (which shows the evolution of both driver electromagnetic wave total actions $N_{0}$, and $N_{s}$, together with their sum $N_{e m}=N_{0}+N_{s}$ ), one see that, while the $N_{0}$-to- $N_{s}$ conversion
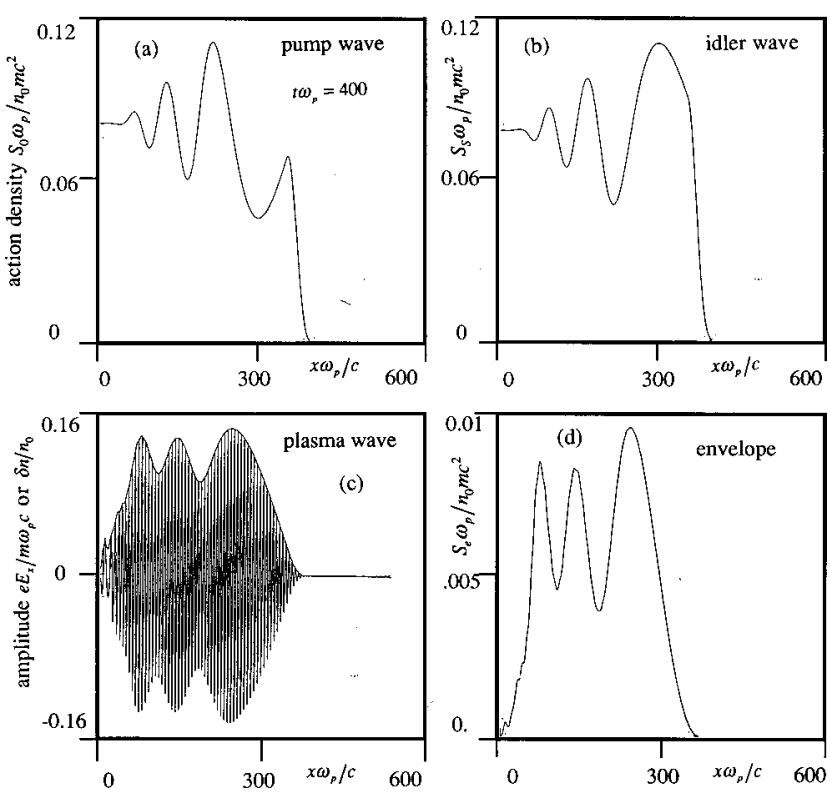

FIG. 9. This is a case of a chirp that is definitely too fast (supercritical in the terminology of Fig. 6) $\left(T \omega_{p}=1.5 \times 10^{5}\right)$. With the same display as for Fig. 7 , shown at the same time $\left(t \omega_{p}=400\right)$ is the spatial behavior of both driver waves and of the corresponding longitudinal field. Note the relatively rapid oscillation of the wave envelopes and the general resemblance to the supercritical and fast results of Fig. 6.

is something like three times the no-chirp result, it is only half that using the stronger chirp. The difference is also striking when one examines Fig. 8(b), where we have plotted [as for Fig. 5(b)] the temporal behavior of $N_{2}=N_{0}+N_{e}+N_{W P}$ and of each quantity taken separately. Although the beat frequency chirping indeed enhances the growth of the plasma wave with respect to the no-chirp result (but not to the level of the stronger chirp result), the energy in trapped particles (proportional to $N_{W P}$ ), is only about a third of that for the stronger chirp, presumably because the plasma wave is less than for the stronger chirp. The $N_{W P}$ is relatively less compared with the $N_{e}$ for this intermediate chirp rate because significant nonlinearity of the extraction and acceleration of the electrons in the hot tail.

Of course, with a slower chirp one could use a longer plasma, but in most cases that one might imagine, obtaining longer plasmas of acceptable uniformity is difficult, so one tries to make the most of plasma length one has. It is for this reason that it is desirable to explore to what level the chirp rate might be raised before some natural limit process manifests itself.

\section{B. Chirped beatwave experiment with a too fast chirp rate}

In this subsection we examine the saturation of the electron plasma wave as a result of using a chirp rate which is too fast. A first series of numerical simulations with the much shorter chirp time of $T \omega_{p}=1.5 \times 10^{5}$ (i.e., a chirp rate 3.33 times faster than our previous fastest at $T \omega_{p}=5.0$ $\times 10^{5}$ ) was carried out and the numerical results are shown in Fig. 9. As was the case for Fig. 7 these curves exhibit, at time $t \omega_{p}=400$, for this very rapid chirp, the spatial behavior 

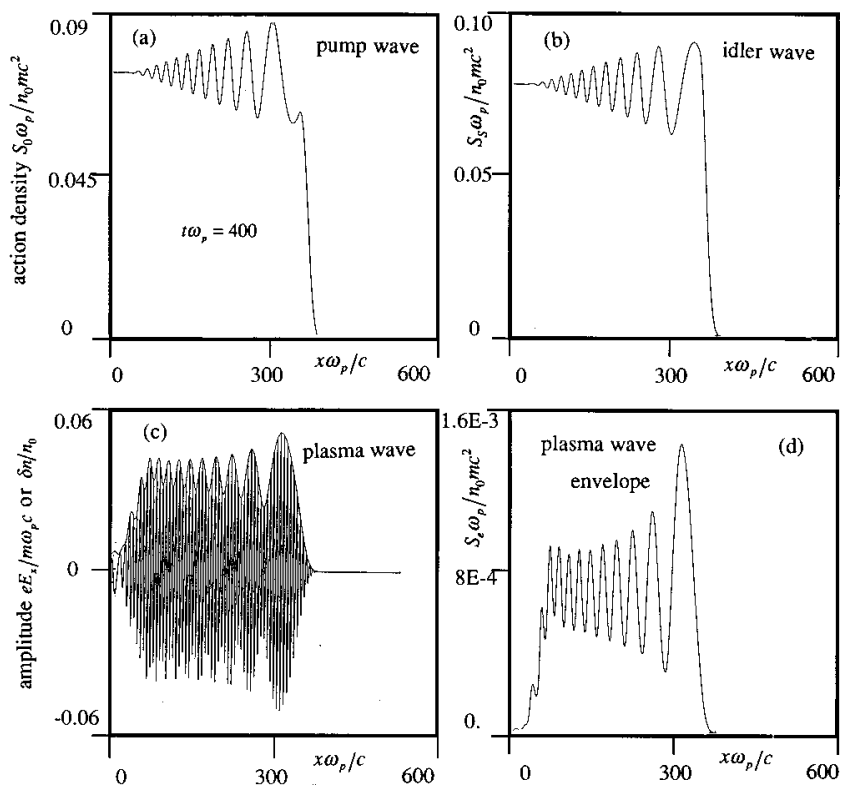

FIG. 10. Numerical results for an even faster chirp rate than for Fig. 9 (corresponding to $T \omega_{p}=8.5 \times 10^{4}$ ) are given in the same format. The rapid oscillations of the envelopes are even more obvious.

of both driver modes and the corresponding longitudinal electric field. To gain some insight into these effects, which might appear puzzling in isolation, let us also consider a second example obtained by decreasing the chirp time even more. We employed an enormously fast chirp rate, 10 times the original value of Figs. 2 and 3, and 3 times the previous one which is already very much too fast (the chirp time now being used is $T \omega_{p}=5.0 \times 10^{4}$ ) and the numerical results for this extremely rapid chirp are summarized in Fig. 10.

When these two sets of results are looked at together, it is clear that they are just what is expected from the chirped RLO model when the chirp rate is set at such a high rate that the plasma oscillations become unlocked from the driving frequency, when one could say that the beatwave drive is "'over-chirped.' As may be seen in Fig. 6 for supercritical and fast chirps, in the RLO model, when the driver is overchirped, the plasma wave amplitude displays a decreasing modulational oscillatory behavior (around a constant value) with time, at a modulation frequency that increases linearly with time. Increasing the chirp rate reduces the amplitude (at least when starting from zero) and reduces the time at which frequency unlocking occurs as well (of course) increasing (i) the rate of change of modulational frequency and (ii) the decrease in modulation amplitude. All these features are present in Figs. 9 and 10, together with the expected effect feedback on the electromagnetic drivers (something one cannot readily include in the RLO model). Just why the amplitude of the beat modulation in the supercritical chirp cases also varies linearly with space in these $H-V$ simulations is not evident.

\section{Critical chirp value: Comparison between the RLO model and the Vlasov plasma}

Because the fully normalized RLO model presents very little load to a computer it was relatively easy to check that the Mora normalization (see Ref. 14) for the RLO model worked and to determine by trial and error the normalized critical chirp value (that value $F_{\text {crit }}^{\prime}$ being, in Mora's units, the highest for which no frequency unlocking occurs) as discussed in Sec. II. For the situation discussed here the translates to a chirp time of $\omega_{p} T_{\text {crit }}=7.3568 \times 10^{5}$, but we find that a somewhat smaller normalized chirp time value of 5.0 $\times 10^{5}$ is still not too short although $1.5 \times 10^{5}$ definitely is so. (A detailed exploration of this topic presented an unacceptable computer burden.) One might guess that the actual critical chirp value (time) might be something like a factor of 2 above (below) the RLO-based estimate.

\section{HIGHLY NONLINEAR REGIME OF PARTICLE ACCELERATION INDUCED BY BEAT FREQUENCY CHIRPING}

In the previous simulations, in order to reduce the computation burden to an acceptable level the driver amplitudes were increased more rapidly than in a comparable experiment such as that at UCLA. In order to see what would happen with a more realistic rise time and with an injected electron beam rather than a high energy tail another simulation was run with the following parameters: $\omega_{0} / \omega_{p} \approx 30$, electron beam injection at $2 \mathrm{MeV}$ energy (but at a number density ratio $n_{b} / n_{0}=10^{-3}$, much higher than of the experiment to avoid problems of numerical truncation), and at a plasma temperature of only $3 \mathrm{keV}$. This gives a value for $k_{e} c / \omega_{p}=1.0094$, which corresponds to $k_{e} \lambda_{D}=0.077$. For a value of $I \lambda^{2} \approx 5.4710^{16} \mu \mathrm{m}^{2} \mathrm{~W} / \mathrm{cm}^{2}$, giving normalized quiver momenta of 0.2 and 0.206 for driver and idler, respectively, the rise time was $120 \mathrm{ps}$ corresponding to $600 \omega_{p}^{-1}$, rather than the $50 \omega_{p}^{-1}$, used in the other simulations just discussed. The chirp rate was chosen to be $T \omega_{p}=8 \times 10^{5}$.

Using the format of Figs 7, 9, and 10, in Figs. 11, 12, and 13 one can see the spatial snapshots of the behavior of the pump, idler, and plasma wave. At first things appear to have gone well and by $t \omega_{p}=400$ in Fig. 11 this slowly-rising chirped case has reached the plasma wave level of the unchirped case with a much more rapid rise in amplitude. However, as shown in Fig. 12, at a normalized time of 500, the normalized electric field $\left(e E_{\max } / m \omega_{p} c\right)$ has reached the very large value of something like 1.2 (at the $x \omega_{p} / c$ value of about 60), just to the right of a very large dip at $x \omega_{p} / c$ of about 30 . This last is clearly associated with a Stokes dip and a pump peak, all of which indicate a very rapid spatial dephasing resulting in energy being returned to the pump. Even more unpleasant is the situation shown in Fig. 13, at the normalized time of 700 , when these localized structures have become more intense with narrower widths and the structures to their left have become dismayingly erratic. Looking at the associated moving-window phase space plots [along the lines of Figs. $12(\mathrm{a}, \mathrm{b}, \mathrm{c})$ ], in the window ahead of this peak-field region, shown in Fig. 14, the acceleration is clearly quite conventional and reaches the healthy normalized momentum value $\left(p_{x} / m c\right)$ of 70 . However, on looking at a window for electrons that have come through this extraordinarily high field region, one sees a much more complicated and relatively badly structured acceleration, one 


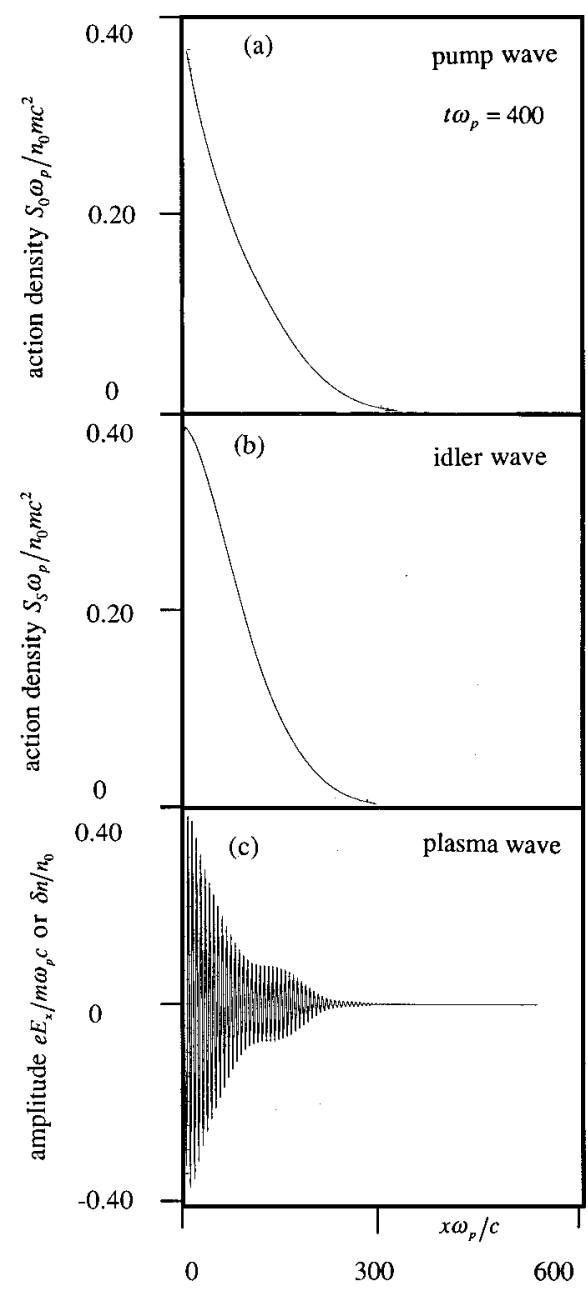

FIG. 11. The results correspond to simulation parameters relevant to the UCLA experiment with $2 \mathrm{MeV}$ electron beam injection and a lower plasma temperature $(3 \mathrm{keV})$ with no hot tail. The realistic driver amplitude rise time is much slower (being $600 \omega_{p}^{-1}$ ) than that for all the previous cases (which used $50 \omega_{p}^{-1}$ ). The conservative chirp rate chosen was that of Fig. 7, being $T \omega_{p}=8.5 \times 10^{5}$. Displays for various times of the spatial behavior of both the electromagnetic driver envelope [pump in (a) and idler in (b)], together with the longitudinal electric field. Given the slow rate of rise of the amplitude the behavior seems quite reasonable.

which would be quite unacceptable for an accelerator. In Fig. 16 energy distributions corresponding to the frames in Figs. 14 and 15 for the times of 700 show the same behavior. (In view of this behavior, it seems to be pointless to discuss the action behavior along the lines used discussing Figs. 5 and 7.)

As expected, the maximum amplitude of the longitudinal fields exceeds the Rosenbluth and Liu limit, given by $e E_{R L} / m \omega_{p} c \approx 0.475$, when we take into account the weak temporal variation of the pump frequency. Nevertheless, a useful comparison with the theoretical value of the relativistic hot plasma wave breaking limit seems doubtful because of the presence of the injected beam electrons accelerated to high energies. However, for what it is worth, we note that the electric field, strong as it is, is nonetheless still well below the relativistic wave-breaking value (now increased because of our lower temperature). We have, in fact, 1.60 $\approx e E_{\max } / m \omega_{p} c<e E_{W B r e l} / m \omega_{p} c \approx 3.27$.

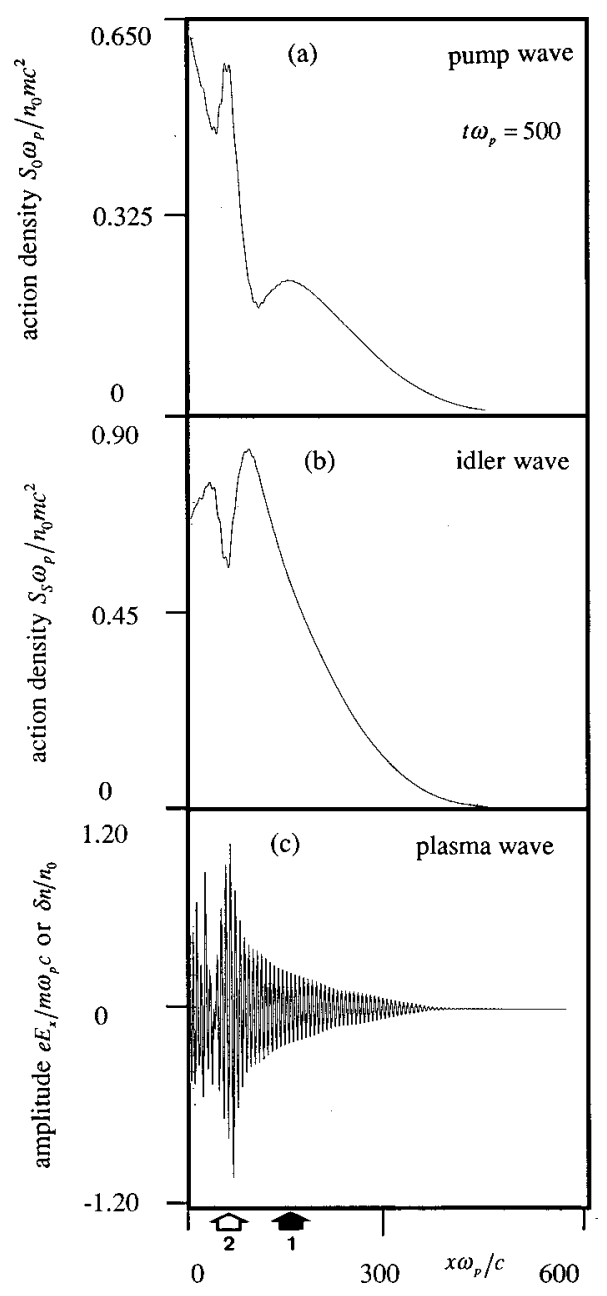

FIG. 12. The same case as Fig. 11, but at a normalized time of 500. A remarkably concentrated field structure is developing with extremely high field values (nearly 1.2) near the entrance, together with puzzling peaks and valleys in the pump and idler action profiles. Note the location of the windows whose phase space snapshots are shown in Fig. 14 (for window 1) and in Fig. 15 (for window 2).

More details of particle acceleration can be found in Fig. 14 and Fig. 15, in which we have represented the time evolution of two different windows moving at the phase velocity of the plasma wave. The spatial location of each moving window throughout the simulation is indicated in Fig. 12(c) by an arrow. Furthermore the gray-shading indicates again the magnitude of the particle distribution function. (We have represented $f$ between $10^{-5}$ and $10^{-4}$ as black for $f \geqslant 10^{-4}$ and white for $f \leqslant 10^{-5}$.) Since the windows are carefully tailored to move at the phase velocity which is very close to $c$, the acceleration structures seen in phase space are quite stationary in this frame, giving thus a time history of the same population of trapped electrons. It proved helpful to draw the limiting trapped orbit trajectory, i.e., the separatrix of particles in the windows at the same time. Two cases are considered here. The first window shown is the right-hand one marked as "1" in Fig. 12, and shown in Fig. 14 at four different times during its evolution. (It was chosen initially at time $t \omega_{p}=400$ at the left-hand side of the plasma box, in a region of a strong electric field close to $e E_{\max } / m \omega_{p} c \approx 0.40$, but this window proves to contain electrons which have not 


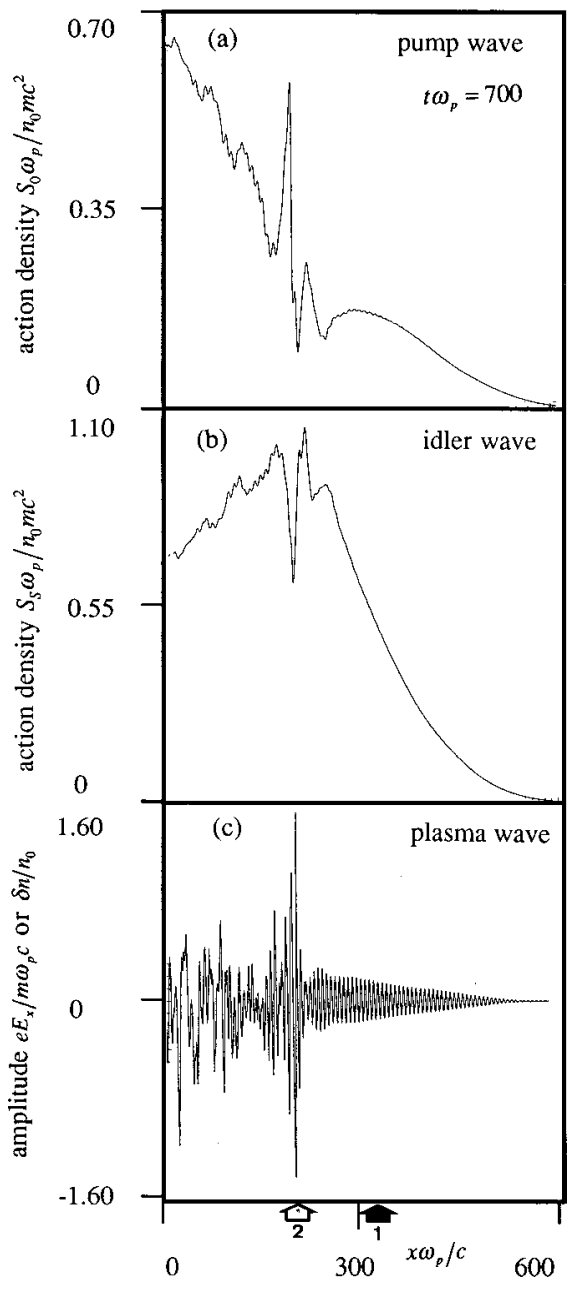

FIG. 13. The same case as Figs. 11 and 12 is continued to a later normalized time (700) and the puzzling behavior of the very strong fields has become even more extreme. The fields are now not what one would accept for a reproducible accelerator.

passed through the anomalous field region once the anomaly became manifest. Thus for this electron population, the acceleration behaves in an adiabatic way, leading to energies up from $10 \mathrm{MeV}$ up to $35 \mathrm{MeV}$. We can notice that, at the end of the window evolution, a beam is formed and decoupling from the plasma bulk. Our second window (marked as "2"' in Fig. 12) shows the complicated phase-space structure associated with the trapped electrons which have passed through this anomalous region of an intense but highly localized field. We see indeed that the phase-space structures which characterize the over-acceleration become more and more stochastic while the field goes on time increasing. However, the phase space structures vortices clearly demonstrate, however, stronger acceleration associated with the beat frequency chirping leading to particle acceleration till $p_{\max } / m c \geqslant 120$, which means to energies that exceed 60 $\mathrm{MeV}$. Further confirmation is provided by the plot in Fig. 16 of the mean distribution function versus the kinetic relativistic energy (given in $\mathrm{MeV}$ units) for both windows, shown at the same time $t \omega_{p}=700$, in Figs. 14 and 15, for the first and second window, respectively. A preliminary estimate of the accelerated particle density indicates that in the range of



FIG. 14. Representation at different times, of the first window (1) of Fig. 12, moving at the phase velocity of the plasma wave, together with the separatrix of particle orbits. This window has been chosen initially in a region of the modest electric field at the beginning of the instability (the instability has not still reached its strongly nonlinear regime). The particle acceleration behaves in an adiabatic way leading to the acceleration level till $p_{x} / m c$ $\geqslant 60$.

10-60 MeV (corresponding to plasma regions of an intense electric field) is roughly 20 times higher in comparison with the population of the first window (accelerated up to 35 $\mathrm{MeV}$ ). By considering an interaction length of $200 \lambda_{0}$ (i.e., $6 \lambda_{e}$ ) for the electric field peak, we can therefore take the effective acceleration length as $2 \mathrm{~mm}$ (for a laser wavelength of $\left.\lambda_{0}=10.275 \mu \mathrm{m}\right)$. Combining this with the numerically measured energy gain of 55-60 MeV implies a peak accelerating gradient of $25-30 \mathrm{GeV} / \mathrm{m}$, which means 10 times the value obtained without a chirp term. Powerful though this is, the anomalous and ill understood nature of this field makes it an unreliable candidate for an accelerator.

As a final remark on this Section, it should be mentioned that an initial suspicion that the development of this intense peak might be an artifact of the $V-H$ code was allayed when it was noticed (i) that a similar phenomenon had been observed in a simple wave-coupling model without nonlinear plasma dynamics (other than the usual mode-coupling terms) and (ii) that the phenomenon could be seen to be beginning in other simulations such as that shown in the plasma wave in Fig. 7.

\section{CONCLUSION AND DISCUSSION}

In this paper numerical simulations using a kinetic Hilbert-Vlasov model have shown that one can increase the plasma wave amplitude above the saturation amplitude limit of Rosenbluth and Liu by time variation ("chirp") of the frequency difference to stay in resonance with the plasma 

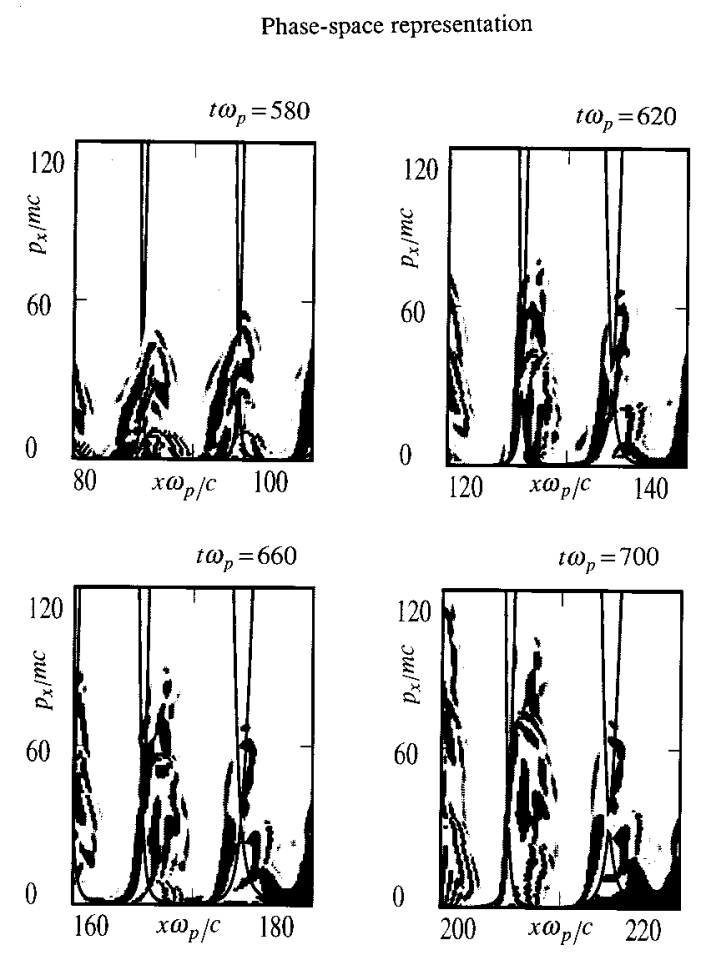

FIG. 15. A similar representation of Fig. 14 but in the case of window 2 in Fig. 12, containing electrons which have come through the anomalous region of highly peaked and very strong electric fields after the strong fields have developed. The particles experience an intense electric field and have been able to reach values for $p_{x} / m c$ as high as 120 . It is clear that the phase-space structures which characterize the over-acceleration have become more and more chaotic.

oscillations. The results are in reasonably consistent with calculations using the Lagrangian oscillator model of Rosenbluth and Liu with "chirp" added. The simulations have shown the appearance of a stronger particle acceleration process induced by the beat frequency chirping in the highly nonlinear regime, leading to a high acceleration gradient of more than $25 \mathrm{GeV} / \mathrm{m}$.

Of considerable importance is the measure of action transferred to fast electrons since this determines accurately the potential efficiency of fast electron production. This action can be directly estimated by considering accelerated particles above the lower separatrix limit. Because the hybrid Vlasov model has essentially no noise, very fine details of the particle distribution function can be easily elucidated, allowing therefore the precise calculation of action transfer. The beat frequency chirping enhances the action transfer to accelerated particles by a factor 2 in comparison with the case without temporal variation of the pump frequency.

For drivers with a rapidly-rising amplitude, chirping seemed to perform very well, but when slower-amplitude rates of rise were used extremely disquieting localized field structures were seen, structures which would pose a threat to well-organized acceleration. Since the nature of this phenomenon is unresolved, it is not clear whether it is unavoidable or not. It would take a significant study to elucidate this point. Unfortunately, this is not a wavelike instability (for which much exists in the way of analytic tools), but rather something like a singular layer which is perhaps self-

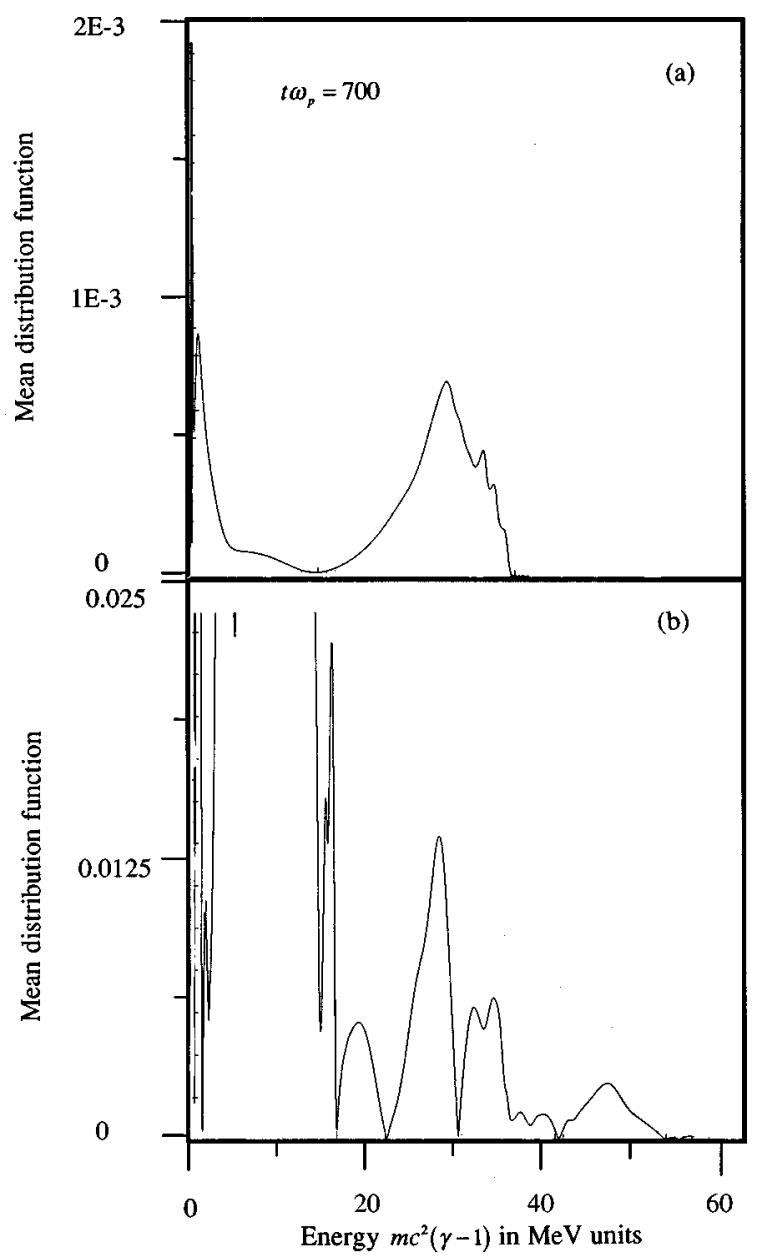

FIG. 16. A display of the mean distribution function versus the relativistic kinetic energy given in $\mathrm{MeV}$ units at the same time for the first and second window in (a) and (b), respectively. The over-acceleration process [in (b)] associated with the introduction of a chirp rate in the simulation leads to energies of $60 \mathrm{MeV}$, corresponding to an accelerating gradient level of $25-30 \mathrm{GeV} / \mathrm{m}$.

organizing. It would be likely difficult to pursuade technologists that these chirp rates are worth pursuing if they seem to lead to accelerators with field singularities.

While the numerical experiments shown in this paper explain clearly the growth of the plasma wave and the limiting due to relativistic detuning and what happens when the frequency is appropriately chirped, the analysis is far from complete, however. Among other aspects, two-dimensional and ion-mediated physics are still to be included. If ion motion and two dimensional motion is included, a variety of instabilities can develop that will limit the amplitude of plasma wave in beatwave experiment and render it incoherent thus unsuitable for sustained particle acceleration. Recent experimental observations (presented in Ref. 14) and also numerical simulations (see, for instance, Ref. 13) are strongly suggestive of the importance of the contribution of the mode-coupling saturation mechanism via the modulational instability in the beatwave experiment. However, the introduction of a beat frequency chirping could be a competitive process to the occurrence of modulational instability.

An another important question is whether the particle acceleration process can be altered by two dimensional spa- 
tial geometry. To check such basic concepts, the much more limited and less demanding spatially two dimensional (2D) periodic Hilbert-Vlasov simulations can be very useful, since in this case, one needs to consider the behavior in time only of the discrete spatial Fourier modes. Thus, it will be possible to take into account the transverse spatial dimension of the electromagnetic driver waves including a complete description of relativistic effects for both the longitudinal and transverse directions. An effort to take into account 2D periodic geometry in the Hilbert-Vlasov code is well in hand and these results should be reported in due course.

\section{ACKNOWLEDGMENTS}

The authors are indebted to the IDRIS center ("Institut du Développement et des Ressources en Informatique Scientifique-Paris-France) for computer time allocation on the Cray-C98 computer. A. Ghizzo and J. Lebas would like to acknowledge the hospitality of the Centre Canadien de Fusion Magnétique of Varennes and the Institut National de la Recherche Scientifique (INRS-Energie et Matériaux) of Varennes (Canada). They are grateful to S. J. Karttunen, RRE Salomaa, and T. J. H. Pättikangas, M. R. Feix, and E. Fijalkow for very helpful discussions.

T. W. Johnston acknowledges the support of the Ministère de l'Education du Québec and of the Canadian National Scientific and Engineering Research Council.
${ }^{1}$ A. Ghizzo, P. Bertrand, J. Lebas, T. W. Johnston, and M. Shoucri, Phys. Plasmas 3, 650 (1996) (Companion paper, often referred to in the text as I).

${ }^{2}$ A. Ghizzo, T. Reveillé, P. Bertrand, T. W. Johnston, J. Lebas, and M. Shoucri, J. Comput. Phys. 11, 356 (1995).

${ }^{3}$ T. W. Johnston, P. Bertrand, A. Ghizzo, M. Shoucri, E. Fijalkow, and M. R. Feix, Phys. Fluids B 4, 2523 (1992).

${ }^{4}$ T. Tajima and J. M. Dawson, Phys. Rev. Lett. 43, 267 (1979).

${ }^{5}$ C. E. Clayton, K. A. Mars, A. Dyson, M. Everett, A. Lal, W. P. Leemans, R. Williams, and C. Joshi, Phys. Rev. Lett. 70, 37 (1993); M. Everett, A. Lal, D. Gordon, C. E. Clayton, K. A. Marsh, and C. Joshi, Nature (London) 368, 527 (1994).

${ }^{6}$ F. Amiranoff, M. Laberge, J. R. Marquès et al., Phys. Rev. Lett. 68, 3710 (1992); F. Moulin, F. Amiranoff, M. Laberge et al., Phys. Plasmas 1, 1318 (1994); F. Amiranoff, D. Bernard, B. Cros et al., Phys. Rev. Lett. 74, 5220 (1995).

${ }^{7}$ M. N. Rosenbluth and C. S. Liu, Phys. Rev. Lett. 29, 701 (1972).

${ }^{8}$ J. P. Matte, F. Martin, N. A. Ebrahim, P. Brodeur, and H. Pepin, IEEE Trans. Plasma Sci. PS-15, 173 (1987).

${ }^{9}$ T. W. Johnston and J. P. Matte, Bull. Am. Phys. Soc. 34, 2024 (1989); F. Charbonneau, J. P. Matte, and T. W. Johnston, ibid. 36, 2365 (1991).

${ }^{10}$ M. Deutsch, B. Meerson, and J. E. Golub, Phys. Fluids B 3, 1773 (1991).

${ }^{11}$ A. Héron and J. C. Adam, Phys. Fluids B 5, 4456 (1993).

${ }^{12}$ T. P. Coffey, Phys. Fluids 14, 1402 (1971); see also W. Kruer, The Physics of Laser Plasma Interactions, Frontiers in Physics (Addison-Wesley, New York, 1988).

${ }^{13}$ Z. M. Sheng and J. Meyer-ter-Vehn, Phys. Plasmas 4, 493 (1997).

${ }^{14} \mathrm{P}$. Mora (unpublished communication). Was available in a 1986 report from the GRECO laser group of the Ecole Polytechnique, Palaiseau, France. 